\title{
Effective vocabulary recall based on episodic and semantic memory types across genders
}

Firoozalizadeh, Mehri $\measuredangle$

Department of Teaching English, Tehran Science and Research Branch, Islamic Azad University, Damavand, Iran (alizadeh.mehri@gmail.com)

Siyyari, Masood

Science \& Research Branch, Islamic Azad University, IAU, Tehran, Iran (m.siyyari@srbiau.ac.ir)

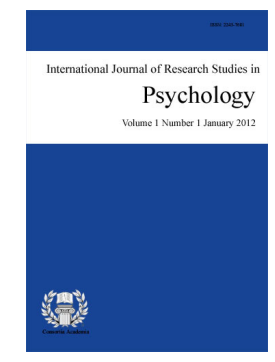

ISSN: 2243-7681 Online ISSN: $2243-769 \mathrm{X}$

OPEN ACCESS

\section{Abstract}

The literature on memory studies shows that gender differences in episodic memory favor women on verbal episodic memory tasks. In contrast, men have been found to have a unique advantage only on episodic memory tasks requiring visuospatial processing. Some studies are also indicative of gender differences favoring women on face recognition and episodic-memory tasks requiring a simultaneous visuospatial and verbal processing (Herlitz \& Rehnman, 2008; Maccoby \& Jacklin, 1974). Given these points, this study was an attempt to compare semantically-oriented and episodically-oriented memory of female and male English learners in terms of learning vocabularies taught to them based on example sentences and definitions. In order to carry out this study, 50 female and male English learners of the intermediate level at Gam-e-Bartar Institute in Iran were selected through an oral placement test and were tested by an episodic memory test (Free Memory Recall Test) and two semantic memory tests (Fluency and Categorical Fluency Test). The participants were taught 37 new English vocabularies by two different strategies, example sentences and definition and were tested upon the newly learnt vocabularies. The data analysis through MANOVA showed a significant difference between women and men in terms of the activation of the episodic memory. Specifically, the results favored women on episodic memory tasks and in recalling positive episodes of their life. Moreover, no significant interaction was found between males and females in terms of their episodically/semantically-learned vocabulary scores indicating that there could be no relation among memory types, gender types, and vocabulary learning.

Keywords: semantic memory; episodic memory; gender; vocabulary recall; second language learning 


\section{Effective vocabulary recall based on episodic and semantic memory types across genders}

\section{Introduction}

The need to conduct new studies has led to numerous researches on teaching and learning second or foreign language vocabularies, focusing on specific strategies of vocabulary teaching, and memory capacity and activities. However the fact that, foreign language learners generally see vocabulary learning as their first priority, the considerable difficulty that they encounter while learning vocabulary is extensively recognized by the language teachers. These problems which have repeatedly pointed out in various learning contexts enthuse the researchers to this field of study. Takac (2008) argued that although psycholinguists have a particular interest in vocabulary development and exploration of the form of the models of vocabulary learning, they ignore the L2 vocabulary literature because it is model-free. On the other hand, applied linguists are mainly involved in the descriptive aspects of the vocabulary and do not account for existing psycholinguistic models of the bilingual lexicon even when this implies an immediate pedagogical significance.

One of the biggest problems with vocabulary learning is that what is learned today is often forgotten tomorrow and almost all people have experienced this problem. Despite the plenty of the researches which have investigated short-term memory, working memory, and procedural memory in explicit learning of vocabulary, the need to conduct further researches to clearly analyze the role of the long-term memory regarding specific components in vocabulary recall is felt. This study is an attempt to examine whether the application of the example sentences and definitions in teaching vocabulary can help female and male learners (to) memorize vocabularies successfully in either semantic or episodic memory, and to investigate how different those vocabularies can be more retrievable from females' memory in comparison with other vocabularies learnt in the specific occasions than in males' memory.

\section{Review of the Related Literature}

Memory on the whole means keeping knowledge somewhere and then retrieving it when it is needed. Long-term memory is the storehouse of information which is relatively permanent. This memory is called the unconscious memory because the information stored in it is largely out of awareness and can be called into working memory to be used when needed. The specialists believe that long-term memory information will be stored in two ways either explicitly or implicitly. The wealth of the findings on implicit and explicit memory is based on the pioneering work of Kolers (1975). Implicit memory, also called "nondeclarative or procedural" (Squire, 1992, p. 233) memory, is nonconscious and requires no conscious awareness. It does not appear to depend on actively recalling earlier learning. On the other hand, explicit memories, sometimes referred to as declarative memory, defined as conscious recollection of episodes, events, items, and information that can easily be verbalized. In order to recall explicit memories which are consciously stored, such as the memory of a past experience, not only should one recall that experience, but he should also recall the condition, date, and place in which that experience has happened in their presence. Two kinds of learning or memory storage (semantic and episodic) have been suggested in explicit memory.

Tulving and Donalson (1972) proposed a theory of memory which draws a line between general knowledge (semantic memory) “... includes knowledge of facts, concepts, moral and social cognition, as well as words and their meaning” (Kasai, Shenton, Salisbury, Onitsuka, Toner, \& Yurgelun-Todd, 2003, p. 983), and memory for experiences (episodic memory) which is the ability to store and recall "temporally-dated episodes and spatiotemporal events" (Tulving \& Donalson, 1972, p. 385) in one's life and a kind of "travel back in time" (Tulving, 2002, p. 2).

As the researches imply, episodic memory is a unique feature of the human being because animals do not 
have a language to test their memories. Schrauf, Pavlenko, and Dewaele (2003) indicated that many of human's memories have been gained through verbal language. While asking to tell a story or movie, and to recall autobiographical memories from his personal life, an individual needs spatiotemporal information about the occurred events (episodic memory of those events, characters, settings, plots), and a language to express them.

Although there were found no gender differences in memory by Maccoby et al. (1974, as cited in Herlitz \& Rehnman, 2008), a number of studies on episodic memory and gender show gender differences favoring women in episodic-memory tasks. There has been proved to be Sex differences in verbal and visuospatial tasks in the abundant studies (e.g., Hyde \& Linn, 1988; Voyer, Voyer, \& Bryden, 1995). Maccoby et al. (1974) pointed out that female learners surpass on verbal tasks and that male learners perform at a higher level than female learners on visuospatial tasks. As Herlitz et al. (2008) stated women surpass on verbal-production task and because of their advantage on such episodic-memory tasks which is the result of their superior verbal-production abilities, there is no wonder that women also surpass when they are questioned to recall events that happened during the past time. According to Herlitz et al. (2008), men can be expected to perform at a higher level than women on episodic memory tasks requiring visuospatial processing due to their better performance on visuospatial tasks.

Herlitz at al. (2008) proposed that throughout the lifetime, verbal episodic-memory tasks grant differences favoring women. The study results on gender differences indicated that women were consistently more successful than men on tasks that stand in need for remembering items that are naturally verbal or can be verbally labeled, such as recalling the words which start with M letter. However, women also did much better on tasks demanding little or no verbal processing, such as unfamiliar odors or faces' recognition. Otherwise, men had advantage on episodic-memory tasks requiring visuospatial processing, such as understanding what an irregular figure looks like when it is rotated in space, and this advantage results in differences favoring men. Herlitz at al. (2008)'s findings suggested that women's episodic-memory advantage can increase or be reversed, depending on the nature of the material to be remembered. In addition, coincidental factors affect the magnitude of the gender differences in episodic memory. For example, if the material is verbal or evokes women's attention (e.g., female faces), women outperform men, whereas men outperform women on visuospatial episodic-memory tasks (e.g., remembering a route).

Following the above-mentioned gaps and related literature, the researchers looked for particular stages of long-term memory which leads the learners' memory to a better recollection of the semantic and episodic information in due context, in which learning has happened. Moreover, the learner's gender as a main factor is considered to see whether learning a second language by investigating episodic and semantic memory precisely would result in a new finding helping the researchers to plan specific syllabus and learning context for female or male learners to achieve success.

\subsection{Research Questions}

Following the purpose of the study, the following research questions are formulated:

Is there any statistically significant difference between males and females in terms of their episodically/semantically-learned vocabulary scores?

\section{Method}

\subsection{Participants}

To meet the objectives of this study, 50 English learners (25 females and 25 males) of the upper-intermediate level at Gam-e-Bartar institute in Iran were selected as the representative sample of the study. The students of upper-intermediate were part of the training program in which a twenty-session course of 90 minutes was undertaking in the English language institute. The students' age ranged from 12 to 30 years old and their 
nationality is Iranian. At the core of the course for this short term research, this study would analyze what was happening with at least 50 English language learners in both definition-based learning of vocabulary and example sentence-based learning.

\subsection{Teacher Vocabulary Test or Test of homogeneity}

In order to carry out this study, two teacher-made vocabulary tests were used. First of all, the researchers selected some advanced vocabularies to provide a piloted test and considered them as the new input teaching to the learners during the treatment. The researchers selected a list of forty-one advanced vocabularies to teach the learners. They were selected from that level for being unknown to the learners in any way. A multiple choice test was designed by the use of these vocabularies. These tests were given to $25 \mathrm{EFL}$ speakers, 8 from elementary level, 9 from intermediate level and 8 from advanced levels. They were asked to choose the best correct answer from among the alternatives. After the analysis of the responses, the internal consistency of the test was very high (alpha $=.88$ ) and 4 items were deleted from the test to avoid too low or too high item facility indices (IF of 19 and $23=0.8$, IF of 35 and $28=0.72$ ). After the deletion of 4 items, a test composed of 37 items was prepared as a posttest to be given to the participants after the treatment.

\subsection{Free Memory Recall \& Fluency and Categorical Fluency Tasks}

The participants were given episodic and semantic memory test, both Free Memory Recall Task as an episodic test and Fluency and Categorical Fluency Task as semantic memory tests. As for episodic memory test, the subjects were given 10 words each denoting three kinds of feeling and memory related to these feelings; positive (wedding), neutral (forest), and negative (separation) words. They were given 30 seconds for each word to write a memory or an episode of their life about that word. The instructor gave the participants a Fluency Test which asked them to write as many names of animals as possible in 30 seconds. The other was a Categorical Fluency Test in which the test takers were asked to write as many names of cities as possible beginning with "M" in 30 seconds. All of these memory tests were held in the participants' L1 to avoid any lack of knowledge and stress on the part of the participants. The treatment program was begun by the instructor as follows.

\subsection{Treatment Materials}

The participants were taught 37 advanced vocabularies in two different categories, one group of vocabulary were considered as episodic vocabulary which had to be taught by example sentences and stories or memories with no absolute definition or synonym, and the second group, called semantic vocabulary, had to be taught by just giving definition or synonym, not giving any example at all.

\subsection{Data Collection Procedure}

The design to conduct this study was Ex-post Facto, with two pretests (a test of memory and a test of vocabulary), a treatment for all learners, as well as a posttest. A thirty-seven-item true/false vocabulary test was used to check whether the vocabularies which were planned to teach were new to learners or not. The participants were asked to check any item which seemed known to them and write the definition of that vocabulary. Based on the analysis of this test, the learners who knew some of the new vocabularies were removed from the data analysis. Successively, a validated teacher-made post-test of vocabulary were given to the learners one week after the treatment process to check the impact of the instruction.

\subsection{Data Analysis}

The question analysis commences with running version 16 of SPSS and dividing participants' scores on the semantic and episodic memory into 4 quartiles. The descriptive statics and testing the normality assumption for the data were presented by employing Kolmogorov-Smirnov test to see whether parametric or non-parametric tests should be employed. Then, the relevant mean comparison statistic will be employed to test the null 
Effective vocabulary recall based on episodic and semantic memory types across genders

hypothesis relevant to the research question. Following this, the correlations between most of the variables of this study (i.e., semantic memory test scores, episodic memory test scores, age, episodically-learnt vocabulary scores, and semantically-learnt vocabulary scores) were computed to see if any statistically significant difference could be found between pairs of the variables.

\section{Results}

\subsection{The Results of Analysis Used for Investigating the Research Question}

Is there any statistically significant difference between males and females in terms of their episodically/semantically-learned vocabulary scores?

In order to investigate the answer to this question, the descriptive statistics on the males and females' episodically and semantically-learned vocabulary scores were computed (Table 1), and then the data were tested for the assumption of normality by employing Kolmogorov-Smirnov test, the results of which in Table 2 indicate that only the data for females' semantically-learned vocabulary scores are not normally distributed $(p<.05)$.

Table 1

Descriptive Statistics for Males and Females' Episodically and Semantically-Learned Vocabulary Scores

\begin{tabular}{|c|c|c|c|c|}
\hline & & gender & Statistic & SE \\
\hline \multirow{12}{*}{$\begin{array}{l}\text { episodically-learned } \\
\text { vocabulary }\end{array}$} & \multirow{6}{*}{ male } & $\mathrm{M}$ & 9.3913 & .72160 \\
\hline & & SD & 3.46068 & \\
\hline & & Min & 4.00 & \\
\hline & & Max & 17.00 & \\
\hline & & Skewness & .466 & .481 \\
\hline & & Kurtosis & .013 & .935 \\
\hline & \multirow{6}{*}{ female } & $\mathrm{M}$ & 10.9643 & 1.02027 \\
\hline & & SD & 5.39878 & \\
\hline & & Min & 2.00 & \\
\hline & & Max & 19.00 & \\
\hline & & Skewness & -.151 & .441 \\
\hline & & Kurtosis & -1.248 & .858 \\
\hline \multirow{12}{*}{$\begin{array}{l}\text { semantically-learned } \\
\text { vocabulary }\end{array}$} & \multirow{6}{*}{ male } & $\mathrm{M}$ & 6.6522 & .61151 \\
\hline & & SD & 2.93271 & \\
\hline & & Min & 1.00 & \\
\hline & & Max & 12.00 & \\
\hline & & Skewness & -.157 & .481 \\
\hline & & Kurtosis & -.198 & .935 \\
\hline & \multirow{6}{*}{ female } & $\mathrm{M}$ & 8.9286 & 1.01043 \\
\hline & & SD & 5.34671 & \\
\hline & & Min & .00 & \\
\hline & & Max & 18.00 & \\
\hline & & Skewness & .298 & .441 \\
\hline & & Kurtosis & -1.181 & .858 \\
\hline
\end{tabular}

Table 2

Normality test results for males/females' episodically and semantically-learned vocabulary scores

\begin{tabular}{lllll}
\hline & gender & \multicolumn{2}{l}{ Kolmogorov-Smirnov } & \\
& & Statistic & df & F \\
\hline \multirow{2}{*}{ episodically-learned vocabulary } & male & .169 & 23 & .086 \\
& female & .130 & 28 & $.200^{*}$ \\
\multirow{2}{*}{ semantically-learned vocabulary } & male & .108 & 23 & $.200^{*}$ \\
& female & .172 & 28 & .033 \\
\hline
\end{tabular}

Since the normality test results above showed that the data for females' semantically-learned vocabulary 
scores are not normally distributed, independent samples $t$ test was employed to compare males and females in terms of their episodically-learned vocabulary scores; however, the non-parametric equivalent of independent samples $t$ test, that is Mann-Whitney $U$ was employed to compare males and females in terms of their semantically-learned vocabulary scores.

Table 3 presents the means of males and females' episodically-learned vocabulary scores. Evidently, females are of a higher mean in comparison to males; however, this difference needs to be checked for statistical significance by employing independent samples $t$ test.

\section{Table 3}

Descriptive statistics on males/females episodically-learned vocabulary scores

\begin{tabular}{llllll}
\hline & gender & $\mathrm{n}$ & $\mathrm{M}$ & $\mathrm{SD}$ & SEM \\
\hline episodically-learned & male & 23 & 9.3913 & 3.46068 & .72160 \\
vocabulary & female & 28 & 10.9643 & 5.39878 & 1.02027 \\
\hline
\end{tabular}

Table 4 provides the results of independent samples $t$ test on comparison of the means of the males and females' episodically-learned vocabulary scores, which indicate that the difference between the genders in terms of their episodically-learned vocabulary scores is not significant; $t(49)=-1.207, p>.05$. In other words, the null hypothesis is not rejected, and there is no difference between males and females in terms of their episodically-learned vocabulary scores.

\section{Table 4}

T-test results on comparison of the means of males/females' episodically-learned vocabulary scores

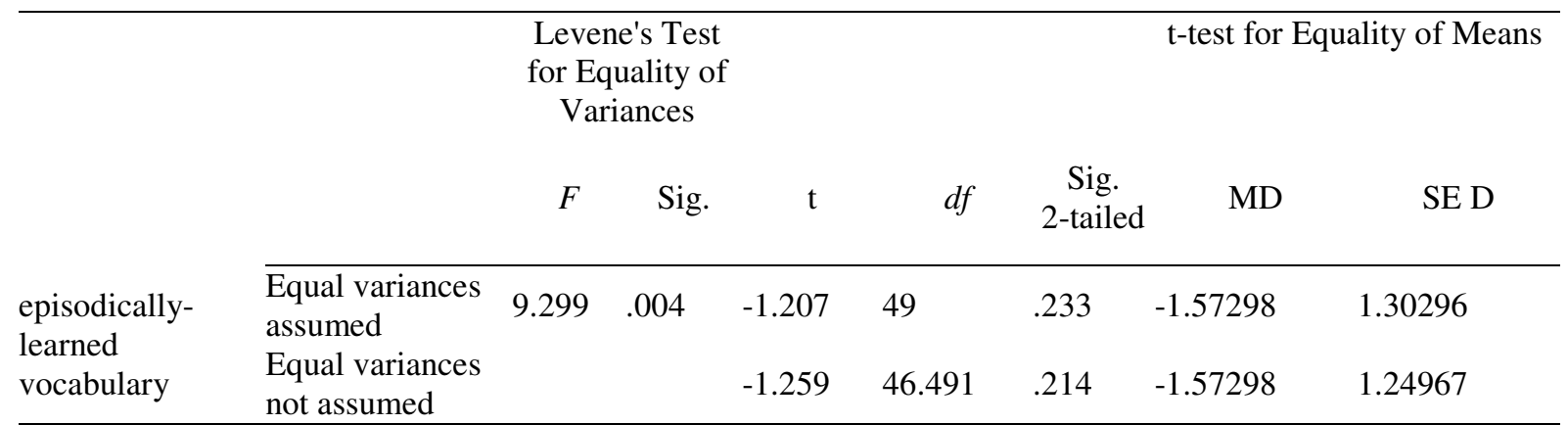

Table 5 presents the males and females' mean ranks in terms of their semantically-learned vocabulary scores. Clearly, females are of a higher mean rank in comparison to males; however, this difference needs to be checked for statistical significance by employing Mann-Whitney U test.

Table 5

Males/females' mean ranks in terms of semantically-learned vocabulary scores

\begin{tabular}{lllll}
\hline & Gender & $\mathrm{n}$ & $\mathrm{M}$ R & S R \\
\hline semantically-learned & male & 23 & 23.35 & 537.00 \\
vocabulary & female & 28 & 28.18 & 789.00 \\
& Total & 51 & & \\
\hline
\end{tabular}

Table 6 provides the results of Mann-Whitney $U$ test on comparison of the means of males and females' semantically-learned vocabulary scores, which indicate that the difference between the genders in terms of their semantically-learned vocabulary scores is not significant; Mann-Whitney $U=261.00, p>.05$. In other words, the null hypothesis is not rejected, and there is no difference between males and females in terms of their semantically-learned vocabulary scores. 
Effective vocabulary recall based on episodic and semantic memory types across genders

Table 6

Mann-Whitney $U$ test results on the means of males/females' semantically-learned vocabulary scores

\begin{tabular}{ll}
\hline & true semantically-learned vocabulary \\
\hline Mann-Whitney U & 261.000 \\
Wilcoxon W & 537.000 \\
$\mathrm{Z}$ & -1.160 \\
Asymp. Sig. (2-tailed) & .246 \\
\hline Note. A Grouping Variable: Gender &
\end{tabular}

Table 7 presents one way ANOVA by taking gender as the main factor in all of the tests. By the analysis of the episodic memory of the females and males, the researchers conclude that there is a significant difference between women and men in terms of the activation of the episodic memory $(p<0.5)$ that is women's episodic memory is higher than men's episodic memory and relatively women did better in recalling positive episodes of their life rather than men.

\section{Table 7}

ANOVA of all tests taking gender as main factor

\begin{tabular}{|c|c|c|c|c|c|c|}
\hline & & SS & $d f$ & MS & $F$ & Sig. \\
\hline \multirow{3}{*}{$\begin{array}{l}\text { Categorical fluency test } \\
\text { (semantic memory test) }\end{array}$} & Between Groups & 1.637 & 1 & 1.637 & .424 & .517 \\
\hline & Within Groups & 262.306 & 68 & 3.857 & & \\
\hline & Total & 263.943 & 69 & & & \\
\hline \multirow[t]{3}{*}{$\begin{array}{l}\text { fluency test (semantic } \\
\text { memory test) }\end{array}$} & Between Groups & 23.692 & 1 & 23.692 & $\begin{array}{l}3.770 \\
.056\end{array}$ & \\
\hline & Within Groups & 427.294 & 68 & 6.284 & & \\
\hline & Total & 450.986 & 69 & & & \\
\hline \multirow[t]{3}{*}{ episodic memory test } & Between Groups & 81.015 & 1 & 81.015 & 8.571 & .005 \\
\hline & Within Groups & 633.275 & 67 & 9.452 & & \\
\hline & Total & 714.290 & 68 & & & \\
\hline \multirow[t]{3}{*}{ neutral episodic score } & Between Groups & 1.827 & 1 & 1.827 & 1.042 & .311 \\
\hline & Within Groups & 117.477 & 67 & 1.753 & & \\
\hline & Total & 119.304 & 68 & & & \\
\hline \multirow[t]{3}{*}{ positive episodic score } & Between Groups & 8.918 & 1 & 8.918 & 7.315 & .009 \\
\hline & Within Groups & 71.934 & 59 & 1.219 & & \\
\hline & Total & 80.852 & 60 & & & \\
\hline \multirow[t]{3}{*}{ negative episodic score } & Between Groups & 1.650 & 1 & 1.650 & 1.346 & .251 \\
\hline & Within Groups & 72.350 & 59 & 1.226 & & \\
\hline & Total & 74.000 & 60 & & & \\
\hline \multirow[t]{3}{*}{ Whole vocabulary test } & Between Groups & 187.110 & 1 & 187.110 & 2.644 & .110 \\
\hline & Within Groups & 3467.635 & 49 & 70.768 & & \\
\hline & Total & 3654.745 & 50 & & & \\
\hline \multirow{3}{*}{$\begin{array}{l}\text { true episodically-learned } \\
\text { vocabularies }\end{array}$} & Between Groups & 31.244 & 1 & 31.244 & 1.457 & .233 \\
\hline & Within Groups & 1050.443 & 49 & 21.438 & & \\
\hline & Total & 1081.686 & 50 & & & \\
\hline \multirow{3}{*}{$\begin{array}{l}\text { true semantically-learned } \\
\text { vocabularies }\end{array}$} & Between Groups & 65.435 & 1 & 65.435 & 3.336 & .074 \\
\hline & Within Groups & 961.075 & 49 & 19.614 & & \\
\hline & Total & 1026.510 & 50 & & & \\
\hline
\end{tabular}




\section{Discussion of the Results and Conclusions}

One point worth mentioning is that episodically-taught vocabularies were learned and recalled better and faster than semantically-taught vocabularies in short-term memory of the learners especially by females while they recalled those vocabularies at the same rate in long term recollection and this is in accordance with Hulstjn's (1992, as cited in Laufer \& Hulstjn, 2001) idea that words are learnt better by providing learners with appropriate context than giving them just some synonyms. This may be the result of higher activation of episodic memory of female learners which ranked higher than men while recalling positive episodes of their life.

As it is mentioned in a research, Herlitz at al. (2008) concluded that there is a significant difference between episodic memory of males and episodic memory of females in a way women consistently outperform men on tasks that demand remembering items that are naturally verbal, and do better on tasks requiring little or no verbal processing, such as recognition of unfamiliar odors or faces. On the other hand, men had the advantage on episodic-memory tasks requiring visuospatial processing. However, vocabulary learning by either method could be of no concern with gender types due to no differentiation between females and males in terms of the activation of episodic and semantic memory. Herlitz at al. (2008)'s findings suggest that women's episodic-memory advantage can develop or be overturned, depending on the nature of the material to be remembered. The types of vocabulary which were taught were of no relation with having any priming effect with a particular gender and there was no bias over any gender in accordance with vocabulary types or family. Although our tests were the same for males and females and the episodic memory test was a kind of remembering or recalling an episode of life (including all verbal, recognition, and visuaspatial episodic memory tasks), our data analysis showed there is a significant difference between women and men in terms of activation of episodic memory that is, women's episodic memory is higher than men's episodic memory, and relatively women did better in recalling positive episodes of their life rather than men. Nevertheless, this minute superiority of female learners over male learners was not effective on the better learning of vocabularies by example-based method.

Although women's episodic memory is higher than men's episodic memory, the results of independent samples $t$ test on comparison of the means of males and females' episodically-learned vocabulary scores indicate that the difference between the genders in terms of their episodically-learned vocabulary scores is not significant; $t(49)=-1.207, p>.05$. In other words, the there is no difference between males and females in terms of their episodically-learned vocabulary scores; however, they may have some advantages over each other in different parts of materials to be remembered in episodic memory.

\subsection{Implications for Teacher Training and Administration}

Due to the natural distinctiveness of learning mechanism in both genders, and their natural preferences in vocabulary learning, co-education would possibly be more helpful in vocabulary learning. Simply put, both genders scaffold each other's learning by providing distinctive strategies for learning new words and they indirectly peer-review their performance by giving and receiving feedbacks.

\subsection{Implications for Materials Development, and Practice}

Based on the above mentioned implications policy makers, in part, would ask for the revision of the material development. In materials development, equal distribution of words, irrespective of their frequency of use, should be based on the abstractness of words and their proper applicable strategies. Words should be equally distributed across lessons according to both strategies to make both equally essential for learning new words, in accordance with the mutual impact and interplay of episodic and semantic memory.

\subsection{Final Remarks}

By doing this research study to investigate the correlation between episodic and semantic memory and 
specific vocabulary strategies, it is hoped that some contribution is made to the development of language teaching considering gender types. Besides, it is believed that this study covered a narrow scope of long-term memory and vocabulary recall, and other researchers and interested students are recommended to carry out related studies to push the frontiers of knowledge in this regard.

\subsection{Suggestions for Further Research}

Based on the above mentioned implications, one point worth studying is the nature of words; that is, some words by their nature are much more inclined to be learnt episodically while other words seem to enjoy semantic treatment. Abstract words primarily need to be explicitly defined on their possible facets, and subsequently need to be concretized through exemplification. In contrast, if such artificial dichotomization seems sound, action verbs can demonstrate their facets without explicit depiction of notions through definition.

\section{References:}

Herlitz, A., \& Rehnman, J. (2008). Sex differences in episodic memory. Association for Psychological Science, $17(1), 52-56$

Hyde, J. S., \& Linn, M. C. (1988). Gender differences in verbal ability: A meta-analysis. Psychological Bulletin, 104(1), 53-69. http://dx.doi.org/10.1037/0033-2909.104.1.53

Kasai, K., Shenton, M. E., Salisbury, D. F., Onitsuka, T., Toner, S. K., \& Yurgelun-Todd, D. (2003). Differences and similarities in insular and temporal pole MRI gray matter volume abnormalities in first-episode schizophrenia and affective psychosis. Arch Gen Psychiatry, 60(11), 1069-77. http://dx.doi.org/10.1001/archpsyc.60.11.1069

Kolers, P. A. (1975). Memorial consequences of automatized encoding. Journal of experimental psychology: Human learning and memory, 1(6), 689-701. http://dx.doi.org/10.1037/0278-7393.1.6.689

Laufer, B., \& Hulstijn, J. H. (2001). Incidental vocabulary acquisition in a second language: The construct of task-induced involvement. Applied Linguistics, 22(1), 1-26. http://dx.doi.org/10.1093/applin/22.1.1

Maccoby, E. E., \& Jacklin, C. N. (1974). The psychology of sex differences. Stanford: Stanford University Press.

Schrauf, R., Pavlenko, A., \& Dewaele, J. M. (2003). Bilingual episodic memory: An introduction. International Journal of Bilingualism, 7(3), 221-233. http://dx.doi.org/10.1177/13670069030070030101

Squire, L. R. (1992). Declarative and non-declarative memory: Multiple brain systems supporting learning and memory. Journal of Cognitive Neuroscience, 4(3), 232-243. http://dx.doi.org/10.1162/jocn.1992.4.3.232

Takac, V. P. (2008). Vocabulary learning strategies and foreign language acquisition. Toronto: Multilingual Matters LTD

Tulving, E. (1983). Elements of episodic memory. New York: Oxford University press.

Tulving, E. (2002). Episodic memory: From mind to brain. Annual Review psychology, 53(1), 1-25. http://dx.doi.org/10.1146/annurev.psych.53.100901.135114

Tulving, E., \& Donalson, W. (1972). Organization of memory. New York and London: Academic Press.

Voyer, D., Voyer, S., \& Bryden, M. P. (1995). Magnitude of sex differences in spatial abilities: A meta-analysis and consideration of critical variables. Psychological Bulletin, 117(2), 250-270.

http://dx.doi.org/10.1037/0033-2909.117.2.250 
Firoozalizadeh, M. \& Siyyari, M. 